\title{
The prognostic value of PKM2 and its correlation with tumour cell PD-L1 in lung adenocarcinoma
}

\author{
Chang-Ying Guo ${ }^{1,2,{ }^{\dagger}}$, Qian Zhu ${ }^{4+}$, Fang-Fang Tou ${ }^{2 \dagger}$, Xiao-Ming Wen ${ }^{2}$, Yu-Kang Kuang ${ }^{2}$ and $\mathrm{Hao} \mathrm{Hu}^{2,3^{*}}$
}

\begin{abstract}
Background: The prognostic value of PKM2 and its correlation with tumour cell PD-L1 in lung adenocarcinoma (LUAD) is unclear.

Methods: A total of 506 lung adenocarcinoma samples from The Cancer Genome Atlas (TCGA) dataset and 173 LUAD tumour tissues from Jiangxi Cancer Hospital were used to analyse the correlation between PKM2 and PD-L1 expression. We further established a stable LUAD cell line with PKM2 knockdown and confirmed the association via Western blotting and flow cytometry analysis. Moreover, the prognostic values of PKM2 and PD-L1 were evaluated by the Kaplan-Meier method and Cox proportional hazards models.
\end{abstract}

Results: Based on the above two large cohorts, we found that PKM2 was significantly positively associated with PD-L1 expression ( $r=0.132, P=0.003$ and $r=0.287, P<0.001$, respectively). Subsequently, we found that PKM2 knockdown substantially inhibited PD-L1 expression in the A549 LUAD cell line. Moreover, survival analysis showed that higher expression of PKM2 was correlated with significantly shorter overall survival (OS) and disease-free survival (DFS) in lung adenocarcinoma patients $(P<0.001$ and $P=0.050$, respectively). Subgroup analysis showed that lung adenocarcinoma patients who expressed high PKM2 and PD-L1 levels experienced the poorest OS and DFS. Additionally, multivariate analysis suggested that high PKM2 and PD-L1 expression was an independent prognostic indicator for worse OS and DFS ( $H R=1.462, P<0.001$ and $H R=1.436, P=0.004$, respectively).

Conclusions: Our results demonstrated that PKM2 regulated PD-L1 expression and was associated with poor outcomes in lung adenocarcinoma patients.

Keywords: Lung adenocarcinoma, Pyruvate kinase M2, Programmed death ligand 1, Prognostic factor

\section{Background}

Non-small-cell lung cancer (NSCLC) accounts for more than $85 \%$ of lung cancer cases, with approximately two-thirds of NSCLC patients presenting at an advanced stage [1]. Although molecular targeted treatment for NSCLC has improved clinical outcomes for patients with amenable mutations [2-4], only a fraction of patients have these mutations, and acquired resistance to targeted therapies frequently occurs [5]. Hence, the identification of

\footnotetext{
* Correspondence: qianhe89513@163.com

${ }^{\dagger}$ Chang-Ying Guo, Qian Zhu and Fang-Fang Tou contributed equally to this work.

${ }^{2}$ Department of Thoracic Surgery, Jiangxi Cancer Hospital, No. 519 Beijing East Road, Nanchang 330006, China

${ }^{3}$ Department of Thoracic Surgery, Ji'an Central Hospital, Ji'an 343000, China Full list of author information is available at the end of the article
}

new and efficient therapeutic targets for NSCLC remains an unmet need.

Pyruvate kinase isoform M2 (PKM2), a metabolic enzyme that catalyses the later steps of glycolysis, plays a key role in regulating metabolic activities in cancer cells and is necessary for tumour growth [6]. In addition to its known role as a metabolic enzyme, PKM2 can act as a signalling modulator in cancer development and progression $[7,8]$. Previously, we identified that elevated expression of PKM2 is involved in cell proliferation and tumour formation in CD44 ${ }^{+}$A549 lung adenocarcinoma stem cells [9]. More importantly, we found that enhanced PKM2 expression contributes to stress resistance and therapeutic resistance in $\mathrm{CD} 44^{+}$A549 lung adenocarcinoma stem cells, suggesting PKM2 as a potential target for lung adenocarcinoma

(c) The Author(s). 2019 Open Access This article is distributed under the terms of the Creative Commons Attribution 4.0 International License (http://creativecommons.org/licenses/by/4.0/), which permits unrestricted use, distribution, and 
therapy [9]. Recently, Zhang et al. demonstrated that PKM2 interacts with intracellular suppressor of cytokine signalling 3 to decrease ATP production and impair the antigen-presenting abilities of dendritic cells in tumours [10]. Another study suggested that PKM2 regulates hypoxia-inducible factor $1 \alpha$ (HIF-1 $\alpha$ ) activity, interleukin-1 $\beta$ (IL-1 $\beta$ ) induction and IL-10 production, thus promoting the inflammatory response [11]. Overall, these studies indicated that PKM2 could act as a modulatory effector on immune and inflammatory responses.

Interestingly, Eva M. Palsson-McDermott et al. found that PKM2 regulated immune checkpoint PD-L1 expression on tumour and immune cells in the CT26 colon carcinoma animal model [12]. However, the potential effects of PKM2 in regulating PD-L1 expression in lung adenocarcinoma remain unclear. Considering that the targeting of programmed death ligand 1 (PD-L1) has shown promise in patients with advanced NSCLC [1315], we evaluated the association between PKM2 and PD-L1 in lung adenocarcinoma. Recently, Sun et al. evaluated the potential prognostic value of PKM2 in lung adenocarcinoma [16]. Nevertheless, there were several limitations in their study. First, the cohort was relatively small with only 65 patients, and approximately half of patients $(47.6 \%)$ were diagnosed with stage IV disease. Moreover, the authors conducted univariate analysis of recurrence-free survival rather than multivariate analysis of overall survival, which prevented the data from supporting the conclusions drawn. Thus, the prognostic effect of PKM2 expression in lung adenocarcinoma patients remained unclear.

In the present study, which encompasses two large independent cohorts, we found a positive correlation between PKM2 and PD-L1 expression in lung adenocarcinoma patients. Subsequently, we found that the knockdown of PKM2 significantly reduced the total and surface protein levels of PD-L1. Finally, we evaluated the prognostic effect of these two proteins in lung adenocarcinoma. Overall, our findings identified a novel mechanism for the metabolic control of immune escape via the regulation of PD-L1 expression in lung adenocarcinoma. These results, along with previous findings regarding the role of PD-L1 in regulating tumour cell metabolism in tumours [17], provide a rationale for combining PKM2 targeting with PD-L1 inhibitors in the treatment of lung adenocarcinoma.

\section{Methods}

\section{Patients and samples}

This was a retrospective study with a cohort of 173 primary lung adenocarcinoma patients with histologically confirmed and surgically resected tumours at the Thoracic Surgery Department from January 2010-June 2014 at Jiangxi Cancer Hospital (Nanchang, China). Patients who had received neoadjuvant therapy before surgery, including chemotherapy, radiotherapy or immunotherapy, were excluded. All patients underwent lobectomy with additional radical lymph node dissection. Approval was obtained from the Institutional Ethical Board of Jiangxi Cancer Hospital. Verbal informed consent was provided by each patient before their tumour tissue samples were obtained. All procedures involving human samples were conducted according to the Declaration of Helsinki.

\section{Immunohistochemistry (IHC)}

Slides (3 slides per sample) from formalin-fixed paraffin-embedded lung adenocarcinoma tissues were used for the histologic evaluation of PKM2 and PD-L1 expression by IHC. Briefly, 4- $\mu \mathrm{m}$ tissue sections were deparaffinized and subjected to heat-induced antigen retrieval. The slides were incubated overnight at $4{ }^{\circ} \mathrm{C}$ with a rabbit anti-human PKM2 mAb (1:100; D78A4, Cell Signaling Technology, Danvers, MA, USA) or rabbit anti-human PD-L1 mAb (1:100; E1L3N, Cell Signaling Technology). After incubation with horseradish peroxidase-conjugated secondary antibody (Envision $^{\text {Tw }}$ Detection Kit, GK500705, Genetech), diaminobenzidine was used to visualize the staining.

\section{Scoring of PKM2 and PD-L1 expression}

Staining for PKM2 was scored based on a 12-point semi-quantitative scoring system [18, 19]. PKM2 expression was quantified after evaluating both the intensity of the staining $(0=$ negative, $1=$ weakly positive, $2=$ moderately positive, and $3=$ strongly positive) and the distribution of positively stained cells $(0=0 \%, 1=1$ to $10 \%, 2=$ 11 to $50 \%, 3=51$ to $80 \%$, and $4=81$ to $100 \%$ ). The final histological score was determined as the staining intensity $\times$ distribution, with a range from 0 to 12 . The cut-off point was the median (score $<8$, negative expression and $\geq 8$, positive expression).

The scores corresponding to PD-L1 expression on tumour cells (TC-PD-L1) were evaluated as a percentage of positively stained cells in the overall section with tumour cells as described in previous studies [20, 21]. Consistent with many other studies [22, 23], PD-L1 positivity was considered membranous PD-L1 expression in $\geq 5 \%$ of tumour cells. Two independent investigators assessed all slides without prior knowledge of the clinical outcome. When any discrepancies occurred between the two investigators, a consensus was reached by discussion.

\section{Cell line}

The human lung adenocarcinoma A549 cell line was purchased from American Type Culture Collection. Cells were routinely maintained in RPMI-1640 basic medium supplemented with 10\% FBS (Gibco, Life Technologies), $100 \mathrm{units} / \mathrm{mL}$ penicillin and $100 \mathrm{mg} / \mathrm{mL}$ streptomycin at $37^{\circ} \mathrm{C}$ in a humidified atmosphere with $5 \% \mathrm{CO}_{2}$. 
Establishment of stable PKM2 knockdown cells

Lentiviruses expressing PKM2-specific shRNAs (designated PKM2-shRNAs) or the negative control vector (designated scramble-shRNA) were purchased from GenePharma Inc. (Shanghai, China, http://www.genepharma.com). A549 cells were cultured at $1 \times 10^{6}$ cells per well in 6-well plates and infected with PKM2-shRNA and scramble-shRNA lentiviruses in accordance with the manufacturer's instructions. Target sequences with high PKM2 knockdown efficiency (PKM2-shRNA-1: 5' -CCGGCTACCACTTGCAATTATT TGACTCGAGTCAAATAATTGCAAG-TGGTAGTTTTT G-3' and PKM2-shRNA-2: 5' -CCGGGCTGTGGCTCTAG ACACTAAACTCGAGTTTAGTGTCTAGAGCCACAGC TTTTTG-3') and the scrambled sequence without any effect on PKM2 levels (5'-CCGGGAGGCTTCTTATAAG TGTTTACTCGAGTAAACACTTATAAGAAGCCTCTT TTTG-3') were adopted as described previously [6]. After $72 \mathrm{~h}$, stable PKM2 knockdown cells were selected with puromycin $(1 \mu \mathrm{g} / \mathrm{mL})$.

\section{Western blotting}

Cellular PKM2 and PD-L1 protein levels in lung adenocarcinoma cell lines were evaluated in total cell extracts by Western blot analysis. A total of $30 \mu \mathrm{g}$ of protein was fractionated using SDS/PAGE and then transferred onto polyvinylidene fluoride membranes (Immobilon P; Millipore, Bedford, MA, USA). After blocking with 5\% non-fat milk and washing with PBS, the membranes were incubated with rabbit anti-PKM2 mAb (1:1000; D78A4, Cell Signaling Technology, Inc., USA), rabbit anti-PD-L1 mAb (1:1000; E1L3N, Cell Signaling Technology, Inc., USA) and rabbit anti-Glyceraldehyde-3-phosphate dehydrogenase (GAPDH) mAb (1:5000, Cell Signaling Technology, Inc., USA), followed by the corresponding secondary antibodies (1:10000, sc-2004, Santa Cruz Biotechnology). The protein bands were visualized via Chemidoc Touch (Bio-Rad) and quantified relative to GAPDH expression using ImageJ software (NIH, Bethesda, MD, USA).

\section{RNA extraction and qRT-PCR}

Total RNA was isolated from the cell lines using TRIzol reagent (Invitrogen, Carlsbad, CA, USA) and reverse transcribed using a PrimeScript $\mathrm{RT}^{\mathrm{TM}}$ Master Mix (Catalogue no: RR036A; Takara Biotechnology Co., Ltd.), followed by quantitative polymerase chain reaction (qPCR) with GoScript qPCR Master Mix (Promega; Madison, WI, USA) according to the manufacturer's protocol.

The primers for PKM2 were forward 5'-ATTATTTGA GGAACTCCGCCGCCT-3' and reverse 5' - ATTCCGGGT CACAGCAATGATGG -3'.

The primers for GAPDH were forward $5^{\prime}$-AAGG TCATCCCTGAGCTGAA-3' and reverse 5'-TGAC AAAGTGGTCGTTGAGG-3'.
GAPDH was used as an internal control, and the relative levels of mRNA were calculated by the $2[-\Delta \Delta C T]$ method.

\section{Flow cytometry}

A total of $1 \times 10^{6}$ cells were harvested and then stained with PE-conjugated anti-PD-L1 antibody (Cat\# 557924, BD Biosciences) or with the corresponding isotype-matched controls (Cat\# 555749, BD Biosciences) for $30 \mathrm{~min}$ at room temperature. Cells were run on a Gallios Flow Cytometer (Beckman Coulter) and analysed using FlowJo software (TreeStar).

\section{Analysis of TCGA data}

Data regarding mRNA expression, mutational status and survival time from The Cancer Genome Atlas for Lung Adenocarcinoma (TCGA, Provisional) were downloaded from cBioPortal (http://cbioportal.org). RNA-Seq V2 RSEM was utilized to analyse mRNA expression. The cut-off for high/low PKM2 based on mRNA expression for survival analysis was determined by Cutoff Finder. For the CD274 (PD-L1) gene, the cut-off for the PD-L1 high/ low group was recorded according to the positive rate of PD-L1 expression by IHC and then checked for an association with survival. The Mann-Whitney test was used to compare PD-L1 levels between tumours with high PKM2 levels and tumours with low PKM2 levels, as PD-L1 levels were not normally distributed $(P<0.05$ from the Kolmogorov-Smirnov normality test). Moreover, we compared survival differences between the high PKM2/high PD-L1 group and the low PKM2/low PD-L1 group.

\section{Statistical analysis}

Statistical analyses were performed using SPSS 22.0 (IBM Corp., Armonk, NY, USA) and GraphPad Prism 6.07 (GraphPad Software, Inc., La Jolla, CA, USA). Continuous variables were presented as the mean \pm standard deviation and compared by unpaired Student's t tests. The chi-square test or Spearman's rank correlation analysis was used for the analysis of PKM2 and PD-L1 expression levels in tumour cells as categorical or continuous variables, respectively. The log-rank test was applied to test the relationship between mRNA expression and overall survival (OS)/disease-free survival (DFS), and the Kaplan-Meier method was used to depict survival curves. Cox proportional hazards regression models were used to perform univariate and multivariate survival analyses, and only variables whose $P$-values were less than 0.1 in the univariate analysis were included in the multivariate analysis. All statistical significance was set at $P<0.05$ (two-sided).

\section{Results}

Patient clinicopathological features

The baseline clinicopathological characteristics of 173 primary lung adenocarcinoma tissue samples by IHC are listed 
in Table 1 . The average age was 47 y (range $27-67$ y). There were 124 (71.7\%) female patients, and the majority of patients were never smokers $(68.2 \%)$. Fifty-one (29.5\%) patients were diagnosed with stage I, 66 (38.2\%) with stage II, 43 (24.9\%) with stage III, and $13(7.5 \%)$ with stage IV disease. For the major driver mutational status, mutations in epidermal growth factor receptor $(E G F R)$ and Kirsten rat sarcoma viral oncogene homologue (KRAS) were detected in 20 (11.6\%) and $25(14.4 \%)$ patients, respectively. Anaplastic lymphoma kinase $(A L K)$ translocations were found in eight patients (4.6\%) and were mutually exclusive with mutations in EGFR and KRAS. Immunohistochemical analysis of lung adenocarcinoma tumour tissues showed that PKM2 was primarily expressed in the cytoplasm of tumour cells, whereas PD-L1 was expressed both on the membrane and in the cytoplasm of tumour cells (Fig. 1). Considering that PD-L1 is mainly expressed on the cell surface, we only evaluated the membrane expression of PD-L1 and termed it TC-PD-L1. Finally, 65 (37.6\%) cases displayed positive TC-PD-L1 staining (Fig. 2C). We next explored the correlation between PKM2 expression and tumour biology and observed that PKM2 expression was associated with $\mathrm{N}$ classification $(P=0.006)$ and TNM stage $(P=0.005)$; however, no significant correlation was observed between PKM2 expression and T classification $(P=0.114)$, $M$ classification $(P=0.232)$ (Table 1$)$. Nevertheless, no strong relationships were detected between PKM2 expression and other clinicopathological characteristics, including patient age, sex and smoking history.

Table 1 Association between PKM2 expression and clinicopathological features of 173 LUAD patients

\begin{tabular}{|c|c|c|c|c|}
\hline \multirow[t]{2}{*}{ Characteristic } & \multirow{2}{*}{$\begin{array}{l}\text { No. of } \\
\text { patients (\%) }\end{array}$} & \multicolumn{2}{|c|}{ PKM2 expression } & \multirow[t]{2}{*}{$P$-value } \\
\hline & & Low & High & \\
\hline Age (years), median (range) & $47(27-67)$ & & & \\
\hline$\leq 60$ & $149(86.1 \%)$ & 77 (51.7\%) & $72(48.3 \%)$ & 0.080 \\
\hline$>60$ & $24(13.9 \%)$ & $17(70.8 \%)$ & 7 (29.2\%) & \\
\hline \multicolumn{5}{|l|}{ Sex } \\
\hline Male & $124(71.7 \%)$ & $67(54.0 \%)$ & $57(46.0 \%)$ & 0.899 \\
\hline Female & $49(28.3 \%)$ & $27(55.1 \%)$ & $22(44.9 \%)$ & \\
\hline \multicolumn{5}{|l|}{ Smoking history } \\
\hline Never & $118(68.2 \%)$ & $64(54.2 \%)$ & $54(45.8 \%)$ & 0.970 \\
\hline Current/Former & $55(31.8 \%)$ & $30(54.5 \%)$ & $25(45.5 \%)$ & \\
\hline \multicolumn{5}{|l|}{ pT classification } \\
\hline $\mathrm{T} 1$ & $48(27.7 \%)$ & $33(68.8 \%)$ & $15(31.3 \%)$ & 0.114 \\
\hline $\mathrm{T} 2$ & 67 (38.7\%) & $31(46.3 \%)$ & $36(53.7 \%)$ & \\
\hline T3 & $46(26.6 \%)$ & $24(52.2 \%)$ & $22(47.8 \%)$ & \\
\hline $\mathrm{T} 4$ & $12(6.9 \%)$ & $6(50.0 \%)$ & $6(50.0 \%)$ & \\
\hline \multicolumn{5}{|l|}{ pN classification } \\
\hline NO & $100(57.8 \%)$ & $62(62.0 \%)$ & $38(38.0 \%)$ & 0.006 \\
\hline N1 & $47(27.2 \%)$ & $25(53.2 \%)$ & $22(46.8 \%)$ & \\
\hline N2-3 & $26(15.0 \%)$ & 7 (26.9\%) & $19(73.1 \%)$ & \\
\hline \multicolumn{5}{|l|}{ pM classification } \\
\hline MO & $160(92.5 \%)$ & $89(55.6 \%)$ & $71(44.4 \%)$ & 0.232 \\
\hline M1 & $13(7.5 \%)$ & $5(38.5 \%)$ & $8(61.5 \%)$ & \\
\hline \multicolumn{5}{|l|}{ pTNM stage } \\
\hline I & $51(29.5 \%)$ & $38(74.5 \%)$ & $13(25.5 \%)$ & 0.005 \\
\hline$\|$ & $66(38.2 \%)$ & $33(50.0 \%)$ & $33(50.0 \%)$ & \\
\hline III & $43(24.9 \%)$ & 18 (41.9\%) & $25(58.1 \%)$ & \\
\hline IV & $13(7.5 \%)$ & $5(38.5 \%)$ & $8(61.5 \%)$ & \\
\hline Positive EGFR mutational status & $20(11.6 \%)$ & $12(12.8 \%)$ & $8(10.1 \%)$ & \\
\hline Positive KRAS mutational status & $25(14.4 \%)$ & $14(14.9 \%)$ & $11(13.9 \%)$ & \\
\hline Positive ALK translocation status & $8(4.6 \%)$ & $5(5.3 \%)$ & $3(3.8 \%)$ & \\
\hline
\end{tabular}

PKM2, pyruvate kinase M2; EGFR, epidermal growth factor receptor; KRAS, Kirsten rat sarcoma viral oncogene homologue; ALK, anaplastic lymphoma kinase; LUAD, lung adenocarcinoma 

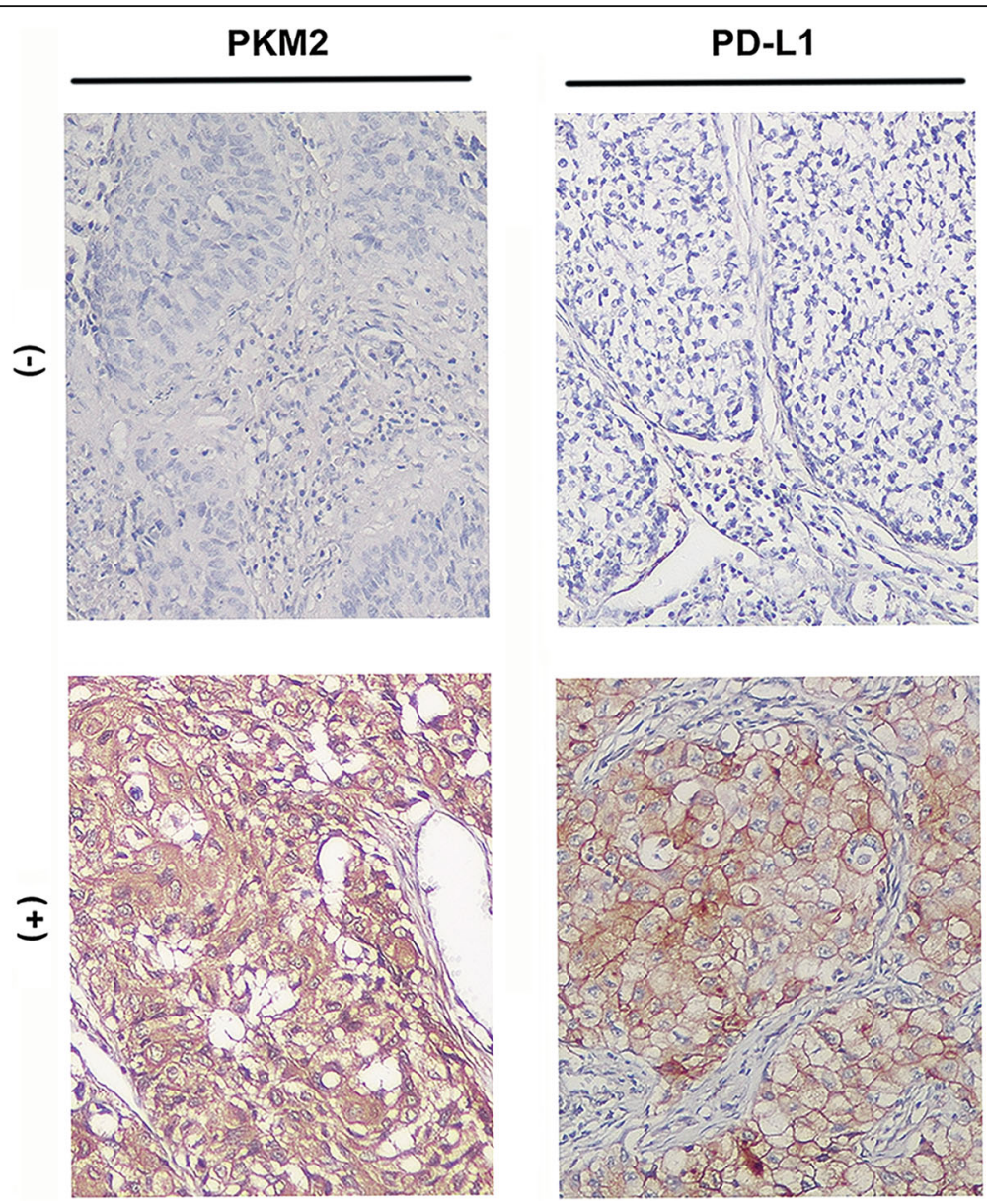

Fig. 1 Representative images of PKM2 and PD-L1 expression in lung adenocarcinoma tumour cells. ( $\times 400$ magnification)

\section{Correlation between PKM2 and PD-L1 in lung adenocarcinoma patients}

First, we evaluated the correlation between PKM2 and PD-L1 based on mRNA expression using TCGA lung adenocarcinoma (LUAD, 506 samples) dataset. A positive correlation between PKM2 and PD-L1 was detected in TCGA LUAD cohort $(\mathrm{r}=0.132, P=0.003)$ (Fig. 2A). Subsequently, we compared PD-L1 expression between the PKM2 low $(n=441)$ and high $(n=65)$ expression groups and found that PD-L1 levels were significantly lower in patients with low PKM2 levels than in patients with high PKM2 levels $(P<0.001)$ (Fig. $2 \mathrm{~B})$. Moreover, we evaluated the correlation between PKM2 and PD-L1 protein expression in 173 primary lung adenocarcinoma tissue samples by IHC. We found that the protein expression of PKM2 was significantly correlated with TC-PD-L1 expression, assessed as categorical variables (Fig. $2 \mathrm{C}, P=0.001$ ). A similar positive association was observed when PKM2 and PD-L1 levels were assessed as continuous variables. Spearman's rank correlation analysis suggested a positive correlation between PKM2 and PD-L1 expression levels $(r=0.287, P<0.001$; Fig. 2D).
Therefore, there is a positive correlation between PKM2 and PD-L1 expression in lung adenocarcinoma patients.

\section{PKM2 regulates PD-L1 expression in the A549 lung adenocarcinoma cell line}

To further understand the relationship between PKM2 and PD-L1 in lung adenocarcinoma tumour cells, we generated stable PKM2 knockdown cells by transfection with PKM2-specific short hairpin RNAs (shRNAs). The levels of PKM2 expression in stable PKM2 knockdown cells were confirmed by both qRT-PCR and Western blotting (Fig. 3A-B). We detected PD-L1 protein expression in stable PKM2 knockdown cells via Western blotting analysis and found that the protein level of PD-L1 was substantially downregulated (Fig. 3C). Moreover, we investigated cell surface PD-L1 protein levels (by flow cytometry) in the PKM2-shRNA and scramble-shRNA groups. The results of flow cytometry analysis revealed that the expression level of PD-L1 was more dramatically reduced in the PKM2-shRNA group compared to that in the scramble-shRNA group (Fig. 3D). 

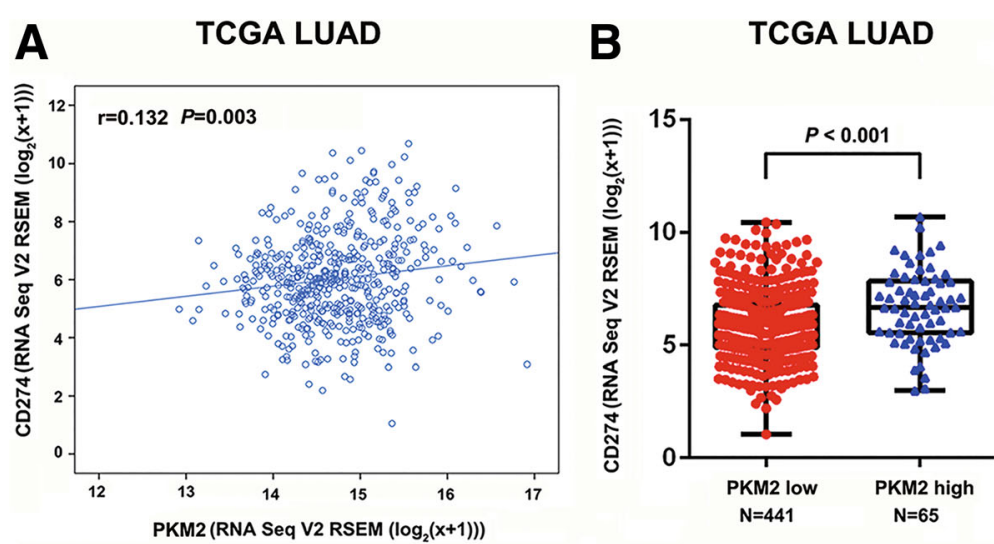

C

\begin{tabular}{llll}
\multicolumn{3}{l}{ Correlation between PKM2 and PD-L1 expression in IHC tissues } \\
\cline { 2 - 4 } & & \multicolumn{3}{c}{ PD-L1 scores } \\
\cline { 2 - 3 } & Negative (\%) & Positive (\%) \\
\hline PKM2 scores & Low & $69(63.9)$ & $25(38.5)$ \\
& High & $39(36.1)$ & $40(61.5)$ \\
\hline$P=0.001$ & & $108(62.4)$ & $65(37.6)$ \\
\hline
\end{tabular}

D

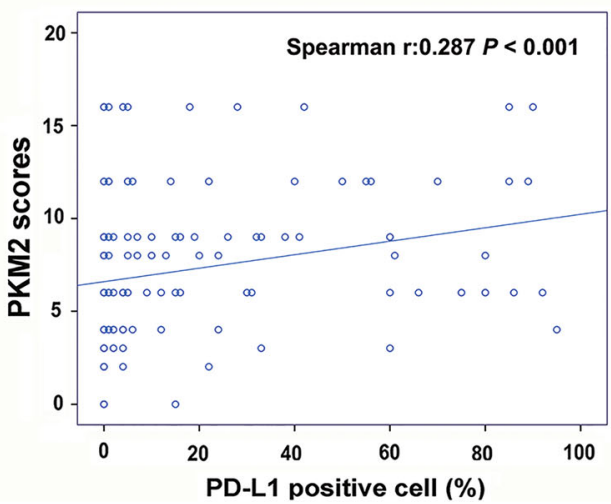

Fig. 2 Correlation of PKM2 with PD-L1 in lung adenocarcinoma. (a) Correlation between PKM2 and PD-L1 mRNA expression in The Cancer Genome Atlas (TCGA) lung adenocarcinoma (LUAD) cohort $(n=506)(P=0.003)$. (b) PD-L1 expression in patients with high PKM2 $(n=441)$ and low PKM2 $(n=65)$ expression $(P<0.001)$. (c) The chi-square test was employed to assess the correlation between PKM2 and PD-L1 expression in samples as categorical variables. (d) Spearman's rank correlation analysis was used to evaluate the strength of the association between PKM2 scores and PD-L1 expression in the tumour cells as continuous variables. ${ }^{*} P<0.05,{ }^{* *} P<0.01$. A box-and-whisker plot was used to represent the data. The box plot represents the first (lower bound), median and third (upper bound) quartiles

\section{Prognostic analysis of PKM2 and PD-L1 expression in} lung adenocarcinoma

Kaplan-Meier survival analysis was performed in TCGA LUAD cohort to compare OS and DFS according to PKM2 and PD-L1 expression. Among the 605 patients included in the survival analysis, positive EGFR mutations, KRAS mutations and $A L K$ translocations were detected in 34 (6.71\%), 74 (14.6\%) and 32 (6.32\%) patients, respectively. To explore the possible relationships between survival and PKM2 and PD-L1 levels, we divided TCGA LUAD cohort into percentiles based on mRNA expression and identified cut-off points for low and high PKM2 at 0.871 and 0.955 for OS and DFS analyses, respectively. Patients with high PKM2 expression had a significantly shorter OS and DFS than patients with low PKM2 expression $(P<0.001$ and $P=0.050$, respectively; Fig. 4A-B). Moreover, the cut-off point was 0.624 and 0.628 for low and high PD-L1 expression for OS and DFS analyses, respectively. We found no statistically significant difference in OS among patients with high vs. low PD-L1 $(P=0.154)$, although patients with high PD-L1 expression seemed to have poorer OS than patients with low PD-L1 expression (Fig. 4C). However, higher PD-L1 expression remained associated with shorter DFS $(P=0.018$, Fig. 4D). Next, we tested whether the combined expression of PKM2 and PD-L1 could improve survival differences between groups (high PKM2 \& high PD-L1 vs. low PKM2 \& low PD-L1) (Fig. 4E-F). Interestingly, we observed that the separation based on OS and DFS between these two groups $(P<0.001$ for 

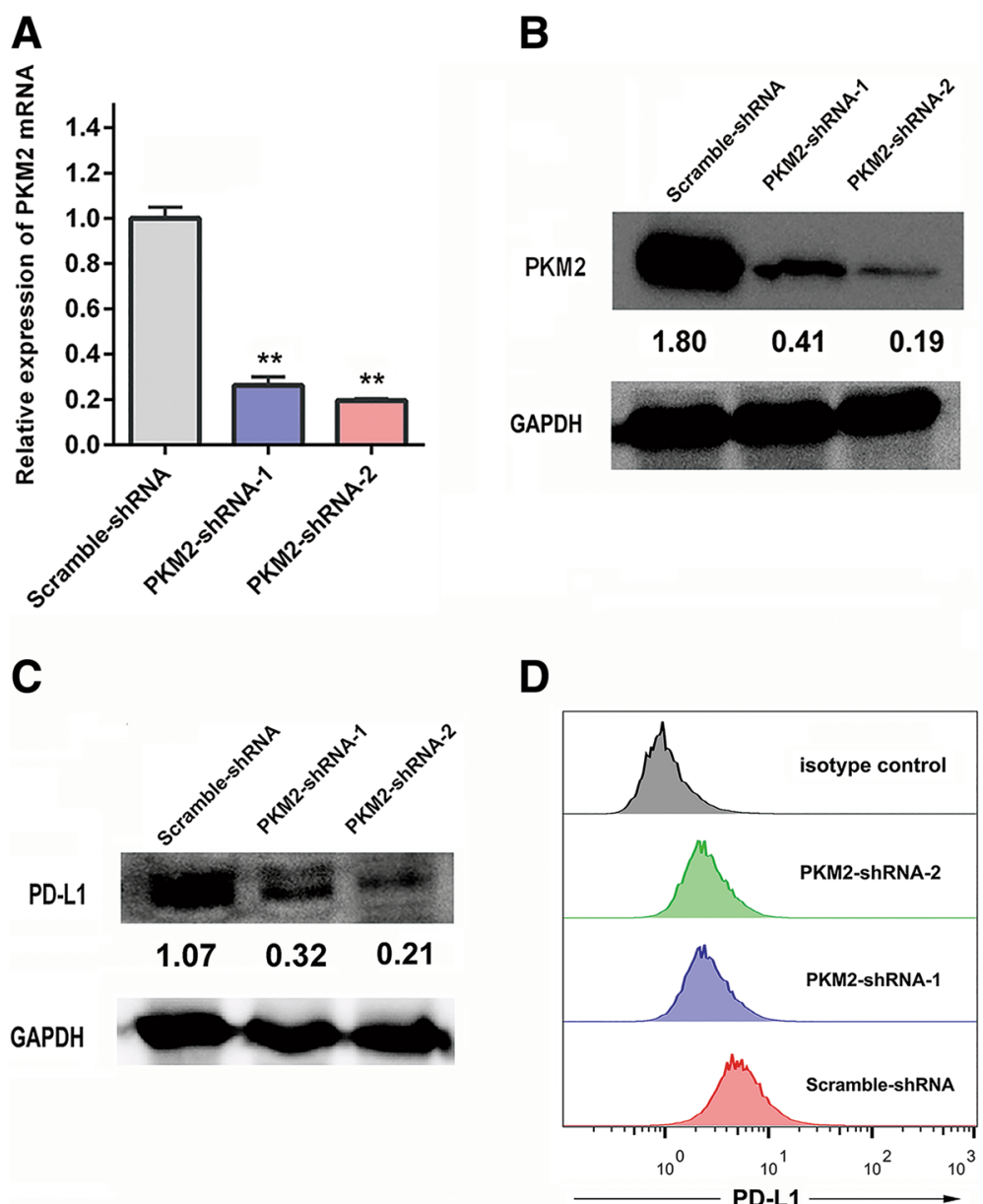

Fig. 3 Regulation of PD-L1 expression by PKM2 in a lung adenocarcinoma cell line. PKM2 expression in A549 lung adenocarcinoma cells transfected with PKM2-shRNA and scramble-shRNA lentiviruses. Seventy-two hours later, the cells were subjected to puromycin selection $(1 \mu \mathrm{g} / \mathrm{mL}) \mathrm{for} 2 \mathrm{weeks}$. Subsequently, the cells were collected for qRT-PCR and protein analysis. Quantification of both qRT-PCR and Western blotting data showing that the knockdown of PKM2 with shRNA-1 and shRNA-2 markedly suppressed PKM2 mRNA levels (a) and total protein expression (b) compared with those with scrambled control. (c) Western blotting of total PD-L1 protein levels in PKM2-shRNA-1-, PKM2-shRNA-2- and scramble-shRNA-transfected lung adenocarcinoma cell lines. (d) Flow cytometric analysis of cell surface PD-L1 levels in PKM2-shRNA-1-, PKM2-shRNA-2- and scramble-shRNA-transfected lung adenocarcinoma cell lines. PKM2-shRNA1, blue zone; PKM2-shRNA2, green zone; scramble-shRNA, red zone; isotype control, grey zone. Unpaired t tests were used to calculate the two-sided $P$-values. ${ }^{*} P<0.05,{ }^{* *} P<0.01$. Error bars in the bar charts represent standard deviation

both) was more apparent than the separation between groups according to PKM2 expression alone (Fig. 4A-B, respectively). To make our results more conclusive, we used random number generators to randomly assign the available set of samples into two groups (a training set and a validation set) in a 1:1 ratio and validated the findings in the two groups (Fig. 5). Finally, we performed univariate and multivariate Cox regression analyses for OS and DFS. All significant clinical factors ( $P$-values less than 0.1 ) in the univariate analysis were included in the multivariate Cox regression analysis (Table 2). As shown in Table 2, high expression of PKM2 and PD-L1 still powerfully and independently predicted poor OS and DFS, indicating that the concomitant PKM2 and PD-L1 expression could be a useful biomarker of response to therapy.

\section{Discussion}

NSCLC is the leading cause of cancer mortality worldwide, and its treatment options have encountered tremendous difficulties [24]. Several studies have revealed an oncogenic role of PKM2 in tumourigenesis and suggested that PKM2 could be a potential target for treating lung adenocarcinoma $[6,16,25,26]$. Interestingly, recent studies reported that PKM2 was involved in the activation of various immune cells, including macrophages [11], dendritic cells [27], monocytes [28] and T cells [29]; however, the possible role of PKM2 in the regulation of the immune checkpoint PD-L1 in lung adenocarcinoma is unknown. In the present study, we demonstrate that PKM2 regulates TC-PD-L1 expression in lung adenocarcinoma using TCGA dataset, tissue samples and cell lines. Considering the importance of 
A

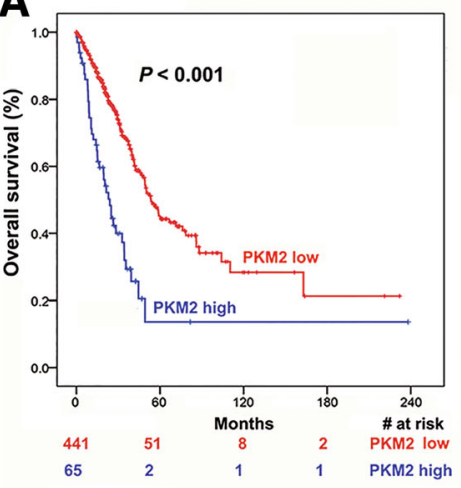

C

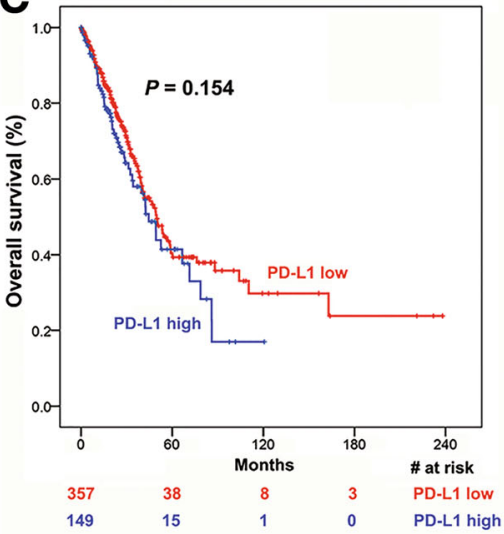

E

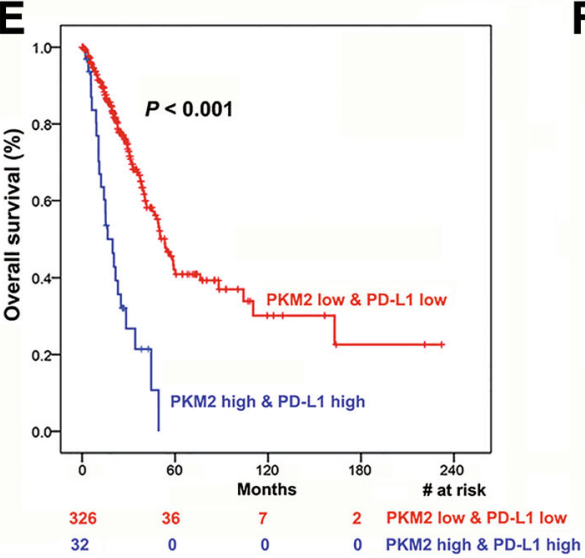

B

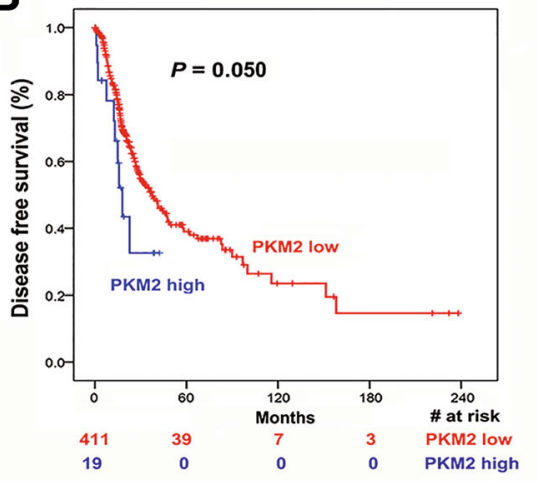

D

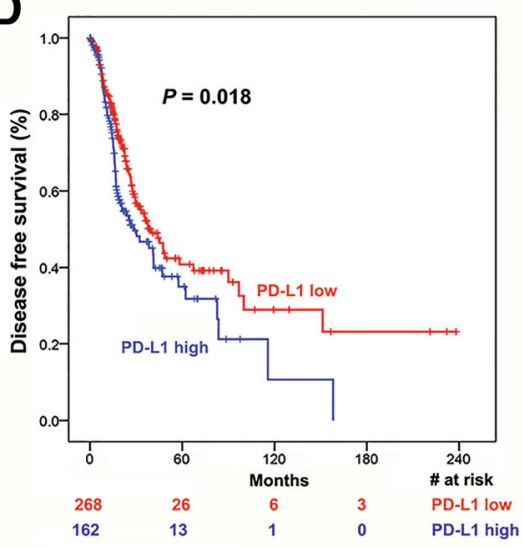

F

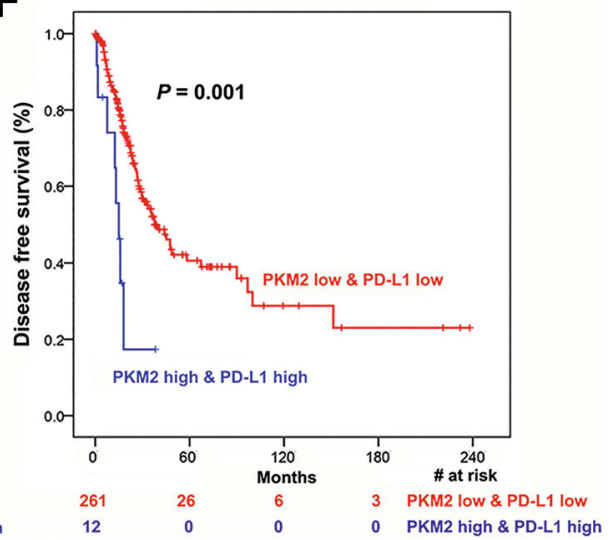

Fig. 4 Prognostic analysis of PKM2 and PD-L1 expression in lung adenocarcinoma. (a-D) Kaplan-Meier analysis of overall survival and disease-free survival according to PKM2 expression (a and $\mathbf{b}$ ) and PD-L1 (c and $\mathbf{d}$ ) expression in TCGA LUAD patient cohorts. (e and $\mathbf{f}$ ) Kaplan-Meier analysis of overall survival and disease-free survival according to the combination of PKM2 and PD-L1 expression in TCGA LUAD patient cohorts. Patients with high PKM2 and high PD-L1 levels had the poorest overall survival (e) and disease-free survival (f) than patients with low PKM2 and low PD-L1 levels or patients with low PKM2 levels alone (a and $\mathbf{b}$ ). The log-rank test was used to determine the association between mRNA expression and overall survival/disease-free survival, and the Kaplan-Meier method was used to generate survival curves. LUAD lung adenocarcinoma; TCGA The Cancer Genome Atlas; Log-rank test, two-sided. The number of patients at risk among these groups at different time points is presented at the bottom of the graphs

PD-L1 expression in NSCLC supported by the clinical benefit of anti-PD-L1 antibodies as single agents in NSCLC patients, these findings further support the investigation of PKM2 as a potential target in lung adenocarcinoma. Moreover, we found that lung adenocarcinoma patients with high PKM2 and PD-L1 expression had the poorest OS and DFS compared with those in patients with low expression of either factor or both factors, indicating that the concomitant PKM2 and PD-L1 expression provides important prognostic information for lung adenocarcinoma patients. This 
A Training set (TCGA-LUAD)

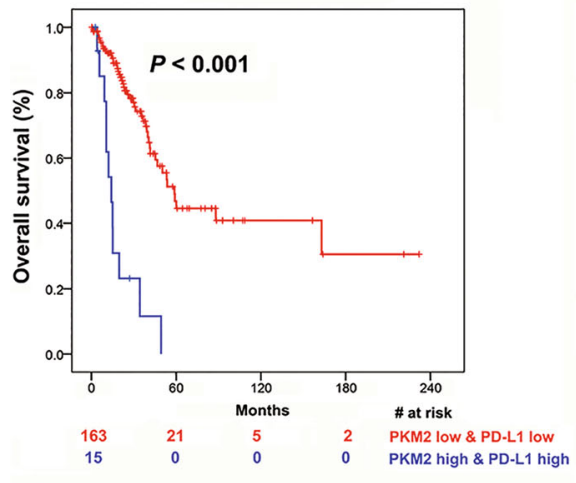

B Validation set (TCGA-LUAD)

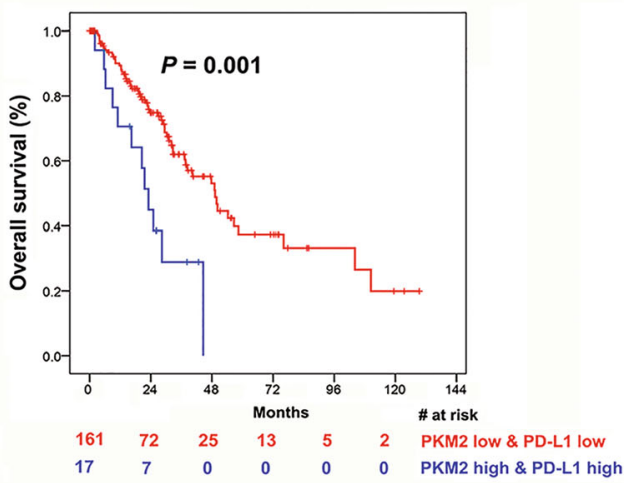

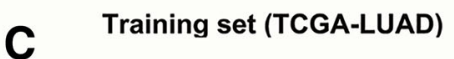

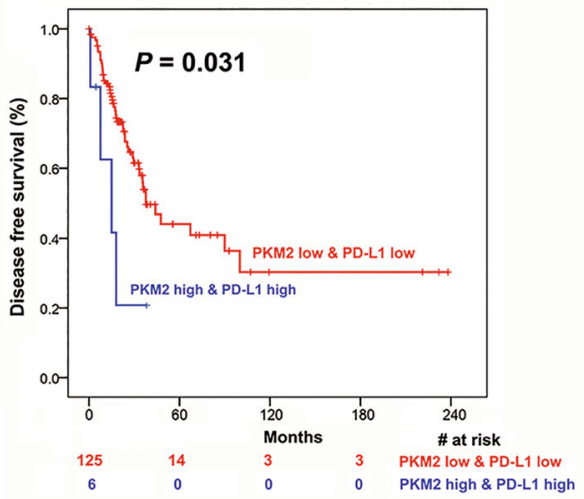

D Validation set (TCGA-LUAD)

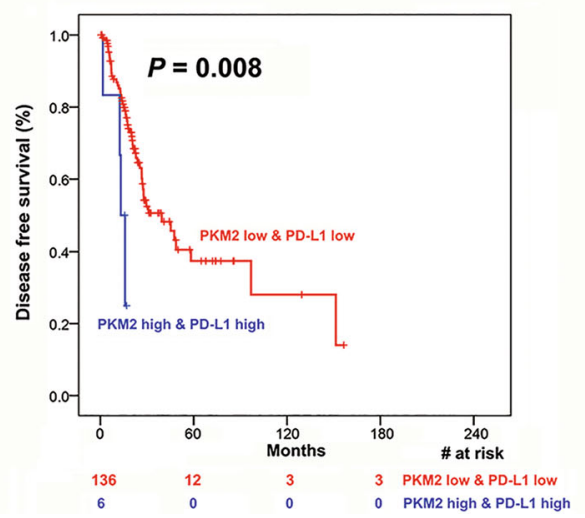

Fig. 5 Association of the combination of PKM2 and PD-L1 expression with prognosis in patients with lung adenocarcinoma in the training and validation cohorts. Kaplan-Meier curves for overall survival and disease-free survival according to the combination of PKM2 and PD-L1 expression in the training ( $\mathbf{a}$ and $\mathbf{b}$ ) and validation ( $\mathbf{c}$ and $\mathbf{d}$ ) lung adenocarcinoma patient cohort from TCGA (TCGA LUAD). The number of patients at risk in the high PKM2 \& high PD-L1 and low PKM2 \& low PD-L1 groups at different time points are presented at the bottom of the graphs

Table 2 Univariate and multivariate Cox regression analyses for OS and DFS in LUAD cohorts

\begin{tabular}{|c|c|c|c|c|}
\hline \multirow[t]{2}{*}{ Variables } & \multicolumn{2}{|l|}{ OS } & \multicolumn{2}{|l|}{ DFS } \\
\hline & $\mathrm{HR}(95 \% \mathrm{Cl})$ & $P$-value & $\mathrm{HR}(95 \% \mathrm{Cl})$ & $P$-value \\
\hline & \multicolumn{4}{|l|}{ Univariate analysis } \\
\hline Age (> 65 vs. $\leq 65)$ & $1.390(0.985-1.963)$ & 0.061 & $1.242(0.852-1.811)$ & 0.260 \\
\hline Sex (female vs. male) & $1.019(0.723-1.437)$ & 0.914 & $1.119(0.770-1.627)$ & 0.555 \\
\hline pT (pT3-4 vs. T1-2 + Tx) & $2.391(1.487-3.843)$ & $<0.001$ & $2.629(1.536-4.501)$ & $<0.001$ \\
\hline $\mathrm{pN}(\mathrm{pN}+\mathrm{vs} . \mathrm{NO}+\mathrm{Nx})$ & $2.698(1.908-3.814)$ & $<0.001$ & $2.236(1.524-3.280)$ & $<0.001$ \\
\hline pM (pM1 vs. M0 + Mx) & $2.049(1.128-3.723)$ & 0.018 & $2.005(0.877-4.584)$ & 0.099 \\
\hline \multirow[t]{2}{*}{ PKM2 high \& PD-L1 high vs. PKM2 low \& PD-L1 low } & $1.581(1.359-1.840)$ & $<0.001$ & $1.481(1.162-1.889)$ & 0.002 \\
\hline & \multicolumn{4}{|l|}{ Multivariate analysis } \\
\hline Age (> 65 vs. $\leq 65)$ & $1.639(1.142-2.353)$ & 0.007 & & \\
\hline pT (pT3-4 vs. T1-2 + Tx) & $1.740(1.057-2.862)$ & 0.029 & $2.178(1.252-3.788)$ & 0.006 \\
\hline$p N(p N+$ vs. $N 0+N x)$ & $2.615(1.823-3.753)$ & $<0.001$ & $2.153(1.461-3.174)$ & $<0.001$ \\
\hline pM (pM1 vs. M0 + Mx) & $1.188(0.633-2.230)$ & 0.592 & $1.658(0.722-3.810)$ & 0.233 \\
\hline PKM2 high \& PD-L1 high vs. PKM2 low \& PD-L1 low & $1.462(1.246-1.715)$ & $<0.001$ & $1.436(1.119-1.843)$ & 0.004 \\
\hline
\end{tabular}


will be useful for the better selection and management of lung adenocarcinoma patients who will benefit from anticancer therapy.

Recent observations have suggested that PKM2 has a dual role in the regulation of immune cell function and antitumour immune responses. On the one hand, PKM2 is imperative for immune cell function and inflammatory responses. For example, PKM2 interacts with HIF- $1 \alpha$ and activates the HIF- $1 \alpha$-dependent transcription of enzymes necessary for aerobic glycolysis, regulating lactate production and high-mobility group box 1 (HMGB1) release in macrophages [30]. Similarly, PKM2 is required for phagocytic activity, the expression of co-stimulatory molecules CD86 and CD80, and the activation of $\mathrm{CD}^{+} \mathrm{T}$ cells by myeloid dendritic cells [27]. On the other hand, PKM2 is involved in the generation of the tumour immune microenvironment. PKM2 enables the metabolic shift from oxidative phosphorylation to aerobic glycolysis and results in increased lactate production, leading to tumour immune evasion in the tumour microenvironment [31, 32]. Other evidence also suggests that cellular metabolism has an integral role in both tumour growth and antitumour immune responses in the tumour immune microenvironment [17, 33]. Overall, these data suggest that PKM2 might contribute to immune regulation in the tumour microenvironment. However, whether PKM2 contributes to tumour immune escape in lung adenocarcinoma is unknown. Here, we show a positive correlation between PKM2 and PD-L1 expression levels in lung adenocarcinoma samples. In addition, knockdown of PKM2 using shRNAs markedly reduced the total protein and cell surface protein levels of PD-L1 in lung adenocarcinoma cells. These findings demonstrate that the regulatory role of PKM2 in human lung adenocarcinoma PD-L1 expression. Our results are broadly consistent with recent findings that PKM2 upregulation contributes to PD-L1 expression in immune cells and CT-26 colon carcinoma cells [12, 34]. Collectively, our findings demonstrate that PKM2 is involved in the regulation of PD-L1 expression in human lung adenocarcinoma cells, indicating the potential of this enzyme as a target in the treatment of lung adenocarcinoma. Moreover, our results complement recent findings involving the role of PKM2 in immune regulation.

A previous study suggested that lung adenocarcinoma patients with higher PKM2 protein expression levels had a significantly shorter recurrence-free survival [16]. We demonstrated that higher PKM2 mRNA levels were associated with shorter OS and DFS in LUAD, further supporting the role of PKM2 expression as a negative prognostic marker in lung adenocarcinoma patients. Previous studies [35-38] have evaluated the prognostic effect of PD-L1 expression in NSCLC, but the results appeared conflicting and inconsistent. In our study, NSCLC patients with higher PD-L1 expression had worse DFS but not OS.
Given the substantially greater prognostic value of multiple biomarkers than of a single biomarker [39], we next investigated whether the combination of PKM2 and PD-L1 expression could improve the prognostic value in lung adenocarcinoma and showed that patients with high PKM2 \& high PD-L1 levels had shorter OS and DFS than patients with low PKM2 \& low PD-L1 levels or patients with high PKM2 or PD-L1 levels alone. Moreover, multivariate Cox regression analyses showed that high expression of PKM2 and PD-L1 was an independent predictor of poor OS and DFS. These findings have important potential clinical applications for identifying patients who will benefit from therapy. However, further study is required to confirm this hypothesis before PKM2 and PD-L1 expression can be used as biomarkers in future clinical applications. Thus far, we have revealed that the combination of PKM2 and PD-L1 is a more powerful prognostic factor in patients with lung adenocarcinoma.

Although this is the first study evaluating the relationship between PKM2 and TC-PD-L1 expression in lung adenocarcinoma, several limitations of our study need to be noted. This study was retrospective in nature, and the sample size was relatively small, which could have biased the results. Hence, a larger independent and prospective study is needed to validate these results in the future. Moreover, other studies are necessary to determine the signalling pathways and factors involved in the regulation of TC-PD-L1 by PKM2 and to distinguish the effects of PKM2 on the tumour immune evasion pathway.

\section{Conclusions}

In conclusion, we demonstrated that PKM2 contributes to TC-PD-L1 expression in lung adenocarcinoma. Moreover, the combination of PKM2 and PD-L1 expression may be a more useful prognostic factor for identifying lung adenocarcinoma patients who might benefit from therapy.

\section{Abbreviations \\ ALK: anaplastic lymphoma kinase; DFS: disease-free survival; EGFR: epidermal growth factor receptor; HIF-1a: hypoxia-inducible factor 1a; HMGB1: high-mobility group box 1; IHC: immunohistochemistry; IL-1 $\beta$ : interleukin-1 $\beta$; KRAS: Kirsten rat sarcoma viral oncogene homologue; LUAD: lung adenocarcinoma; NSCLC: non- small-cell lung cancer; OS: overall survival; PD-L1: programmed death ligand 1; PKM2: pyruvate kinase isoform M2; qPCR: quantitative polymerase chain reaction; TCGA: The Cancer Genome Atlas; TCs: tumour cells}

\section{Acknowledgements}

We would like to thank the reviewers whose comments and suggestions greatly improved this manuscript.

\section{Funding}

This study was supported by grants from the National Natural Science Foundation of China (NO. 81560382), the Jiangxi Province Program of the Preponderant Team Building in Science and Technology Innovation (NO. 20161BCB24011), and the Key Research and Development Program of Jiangxi Province (NO. 20181BBG70017). The funding bodies had no role in the design of the study and collection, analysis, and interpretation of data and in writing the manuscript. 


\section{Availability of data and materials}

The data sets generated and/or analysed during the current study are not publicly available due to confidentiality issues but are available from the corresponding author on reasonable request.

\section{Authors' contributions}

C-Y G and Q Z conducted the immunohistochemistry analysis and drafted the manuscript. F-F T and X-M W collected the tissue specimens and patient information. C-Y G, Q Z and Y-K K performed the statistical analyses. H H conceived of the study and guided the entire project. All authors read and approved the final manuscript.

\section{Ethics approval and consent to participate}

Approval for retrospective analysis of the patient data was obtained from the ethics committee of the Jiangxi Cancer Hospital. Because this is a retrospective study, the ethics committee of Jiangxi Cancer Hospital also waived the need for written consent; verbal consent was obtained from the patients via telephone and documented in the informed consent form if the patient agreed to participate in this study.

\section{Consent for publication}

Not applicable.

\section{Competing interests}

The authors declare that they have no competing interests.

\section{Publisher's Note}

Springer Nature remains neutral with regard to jurisdictional claims in published maps and institutional affiliations.

\section{Author details}

'Department of Thoracic Surgery, Medical College of Nanchang University, Nanchang 330006, China. ${ }^{2}$ Department of Thoracic Surgery, Jiangxi Cancer Hospital, No. 519 Beijing East Road, Nanchang 330006, China. ${ }^{3}$ Department of Thoracic Surgery, Ji'an Central Hospital, Ji'an 343000, China. ${ }^{4}$ Department of Biotherapy, Sun Yat-sen University Cancer Center, Guangzhou 510060, China.

\section{Received: 19 January 2019 Accepted: 25 March 2019}

Published online: 29 March 2019

\section{References}

1. Parsons A, Daley A, Begh R, Aveyard P. Influence of smoking cessation after diagnosis of early stage lung cancer on prognosis: systematic review of observational studies with meta-analysis. BMJ. 2010;340:b5569.

2. Janne PA, Yang JC, Kim DW, Planchard D, Ohe Y, Ramalingam SS, Ahn MJ, Kim SW, Su WC, Horn L, et al. AZD9291 in EGFR inhibitor-resistant nonsmall-cell lung cancer. N Engl J Med. 2015;372(18):1689-99.

3. Shaw AT, Engelman JA. Ceritinib in ALK-rearranged non-small-cell lung cancer. N Engl J Med. 2014;370(26):2537-9.

4. Shaw AT, Ou SH, Bang YJ, Camidge DR, Solomon BJ, Salgia R, Riely GJ, Varella-Garcia M, Shapiro Gl, Costa DB, et al. Crizotinib in ROS1-rearranged non-small-cell lung cancer. N Engl J Med. 2014;371(21):1963-71.

5. Camidge DR, Pao W, Sequist LV. Acquired resistance to TKIs in solid tumours: learning from lung cancer. Nat Rev Clin Oncol. 2014;11(8):473-81.

6. Christofk HR, Vander Heiden MG, Harris MH, Ramanathan A, Gerszten RE, Wei R, Fleming MD, Schreiber SL, Cantley LC. The M2 splice isoform of pyruvate kinase is important for cancer metabolism and tumour growth. Nature. 2008:452(7184):230-3.

7. Tamada M, Suematsu M, Saya H. Pyruvate kinase M2: multiple faces for conferring benefits on cancer cells. Clin Cancer Res. 2012;18(20):5554-61.

8. Li YH, Li XF, Liu JT, Wang H, Fan LL, Li J, Sun GP. PKM2, a potential target for regulating cancer. Gene. 2018;668:48-53.

9. Guo CY, Yan C, Luo L, Goto S, Urata Y, Xu JJ, Wen XM, Kuang YK, Tou FF, Li TS. Enhanced expression of PKM2 associates with the biological properties of cancer stem cells from A549 human lung cancer cells. Oncol Rep. 2017; 37(4):2161-6.

10. Zhang Z, Liu Q, Che Y, Yuan X, Dai L, Zeng B, Jiao G, Zhang Y, Wu X, Yu Y. Antigen presentation by dendritic cells in tumors is disrupted by altered metabolism that involves pyruvate kinase $\mathrm{M} 2$ and its interaction with SOCS3. Cancer Res. 2010;70(1):89-98.
11. Palsson-McDermott EM, Curtis AM, Goel G, Lauterbach MAR, Sheedy FJ, Gleeson LE, van den Bosch MWM, Quinn SR, Domingo-Fernandez R, Johnston DGW, et al. Pyruvate kinase $\mathrm{M} 2$ regulates Hif-1alpha activity and IL-1 beta induction and is a critical determinant of the Warburg effect in LPS-activated macrophages. Cell Metab. 2015;21(2):347.

12. Palsson-McDermott EM, Dyck L, Zaslona Z, Menon D, McGettrick AF, Mills $K H G, O$ 'Neill LA. Pyruvate kinase $M 2$ is required for the expression of the immune checkpoint PD-L1 in immune cells and tumors. Front Immunol. 2017;8:1300.

13. Rittmeyer A, Barlesi F, Waterkamp D, Park K, Ciardiello F, von Pawel J, Gadgeel SM, Hida T, Kowalski DM, Dols MC, et al. Atezolizumab versus docetaxel in patients with previously treated non-small-cell lung cancer (OAK): a phase 3, open-label, multicentre randomised controlled trial. Lancet. 2016.

14. Socinski MA, Jotte RM, Cappuzzo F, Orlandi F, Stroyakovskiy D, Nogami N, Rodriguez-Abreu D, Moro-Sibilot D, Thomas CA, Barlesi F, et al. Atezolizumab for first-line treatment of metastatic nonsquamous NSCLC. N Engl J Med. 2018;378(24):2288-301.

15. Horn L, Mansfield AS, Szczesna A, Havel L, Krzakowski M, Hochmair MJ, Huemer F, Losonczy G, Johnson ML, Nishio M, et al. First-line Atezolizumab plus chemotherapy in extensive-stage small-cell lung Cancer. N Engl J Med. 2018.

16. Sun H, Zhu A, Zhang L, Zhang J, Zhong Z, Wang F. Knockdown of PKM2 suppresses tumor growth and invasion in lung adenocarcinoma. Int J Mol Sci. 2015;16(10):24574-87.

17. Chang CH, Qiu J, O'Sullivan D, Buck MD, Noguchi T, Curtis JD, Chen Q, Gindin M, Gubin MM, van der Windt GJ, et al. Metabolic competition in the tumor microenvironment is a driver of Cancer progression. Cell. 2015;162(6): 1229-41.

18. Kobierzycki C, Pula B, Werynska B, Piotrowska A, Muszczynska-Bernhard B, Dziegiel $P$, Rakus D. The lack of evidence for correlation of pyruvate kinase $\mathrm{M} 2$ expression with tumor grade in non-small cell lung cancer. Anticancer Res. 2014;34(7):3811-7.

19. Lockney NA, Zhang M, Lu Y, Sopha SC, Washington MK, Merchant N, Zhao Z, Shyr Y, Chakravarthy AB, Xia F. Pyruvate kinase muscle isoenzyme 2 (PKM2) expression is associated with overall survival in pancreatic ductal adenocarcinoma. J Gastrointest Cancer. 2015;46(4):390-8.

20. Yang CY, Lin MW, Chang YL, Wu CT, Yang PC. Programmed cell deathligand 1 expression in surgically resected stage I pulmonary adenocarcinoma and its correlation with driver mutations and clinical outcomes. Eur J Cancer. 2014;50(7):1361-9.

21. Mansfield AS, Aubry MC, Moser JC, Harrington SM, Dronca RS, Park SS, Dong H. Temporal and spatial discordance of programmed cell death-ligand 1 expression and lymphocyte tumor infiltration between paired primary lesions and brain metastases in lung cancer. Ann Oncol. 2016;27(10):1953-8.

22. Zhu Q, Cai MY, Chen CL, Hu H, Lin HX, Li M, Weng DS, Zhao JJ, Guo L, Xia JC. Tumor cells PD-L1 expression as a favorable prognosis factor in nasopharyngeal carcinoma patients with pre-existing intratumor-infiltrating lymphocytes. Oncoimmunology. 2017;6(5):e1312240.

23. Mansfield AS, Roden AC, Peikert T, Sheinin YM, Harrington SM, Krco CJ, Dong $\mathrm{H}$, Kwon ED. B7-H1 expression in malignant pleural mesothelioma is associated with sarcomatoid histology and poor prognosis. J Thorac Oncol. 2014;9(7):1036-40

24. Torre LA, Bray F, Siegel RL, Ferlay J, Lortettieulent J, Jemal A. Global cancer statistics, 2012. Ca A Cancer Journal for Clinicians. 2015;65(2):87-108.

25. Parnell KM, Foulks JM, Nix RN, Clifford A, Bullough J, Luo B, Senina A, Vollmer D, Liu J, McCarthy $V$, et al. Pharmacologic activation of PKM2 slows lung tumor xenograft growth. Mol Cancer Ther. 2013;12(8):1453-60.

26. Meng MB, Wang HH, Guo WH, Wu ZQ, Zeng XL, Zaorsky NG, Shi HS, Qian D, Niu ZM, Jiang B, et al. Targeting pyruvate kinase $M 2$ contributes to radiosensitivity of non-small cell lung cancer cells in vitro and in vivo. Cancer Lett. 2015;356(2 Pt B):985-93.

27. Liu C, Zheng M, Wang $T$, Jiang H, Fu R, Wang H, Ding $K$, Zhou Q, Shao Z. PKM2 is required to activate myeloid dendritic cells from patients with severe aplastic anemia. Oxidative Med Cell Longev. 2018;2018:1364165.

28. Huang J, Liu K, Zhu S, Xie M, Kang R, Cao L, Tang D. AMPK regulates immunometabolism in sepsis. Brain Behav Immun. 2017.

29. Lu S, Deng J, Liu H, Liu B, Yang J, Miao Y, Li J, Wang N, Jiang C, Xu Q, et al. PKM2-dependent metabolic reprogramming in CD4(+) T cells is crucial for hyperhomocysteinemia-accelerated atherosclerosis. J Mol Med (Berl). 2018; 96(6):585-600. 
30. Yang L, Xie M, Yang M, Yu Y, Zhu S, Hou W, Kang R, Lotze MT, Billiar TR, Wang $\mathrm{H}$, et al. PKM2 regulates the Warburg effect and promotes HMGB1 release in sepsis. Nat Commun. 2014;5:4436.

31. Warburg O. On the origin of cancer cells. Science. 1956;123(3191):309-14.

32. Romero-Garcia S, Moreno-Altamirano MMB, Prado-Garcia H, Sánchez-García FJ. Lactate contribution to the tumor microenvironment: mechanisms, effects on immune cells and therapeutic relevance. Front Immunol. 2016;7.

33. Loftus RM, Finlay DK. Immunometabolism: cellular metabolism turns immune regulator. J Biol Chem. 2016;291(1):1-10.

34. Deng W, Zhu S, Zeng L, Liu J, Kang R, Yang M, Cao L, Wang H, Billiar TR, Jiang J, et al. The circadian clock controls immune checkpoint pathway in sepsis. Cell Rep. 2018;24(2):366-78.

35. Wu S, Shi X, Sun J, Liu Y, Luo Y, Liang Z, Wang J, Zeng X. The significance of programmed cell death ligand 1 expression in resected lung adenocarcinoma. Oncotarget. 2017:8(10):16421-9.

36. Sepesi B, Nelson DB, Mitchell KG, Gibbons DL, Heymach JV, Vaporciyan AA, Swisher SG, Roszik J. Prognostic value of PD-L1 mRNA sequencing expression profile in non-small cell lung Cancer. Ann Thorac Surg. 2018; 105(6):1621-6.

37. Kim H, Kwon HJ, Park SY, Park Y, Park E, Chung JH. Clinicopathological analysis and prognostic significance of programmed cell death-ligand 1 protein and mRNA expression in non-small cell lung cancer. PLoS One. 2018;13(6):e0198634.

38. Budczies J, Bockmayr M, Denkert C, Klauschen F, Groschel S, Darb-Esfahani S, Pfarr N, Leichsenring J, Onozato ML, Lennerz JK, et al. Pan-cancer analysis of copy number changes in programmed death-ligand 1 (PD-L1, CD274) associations with gene expression, mutational load, and survival. Genes Chromosomes Cancer. 2016;55(8):626-39.

39. Kratz JR, He J, Van Den Eeden SK, Zhu ZH, Gao W, Pham PT, Mulvihill MS, Ziaei F, Zhang H, Su B, et al. A practical molecular assay to predict survival in resected non-squamous, non-small-cell lung cancer: development and international validation studies. Lancet. 2012;379(9818):823-32.

Ready to submit your research? Choose BMC and benefit from:

- fast, convenient online submission

- thorough peer review by experienced researchers in your field

- rapid publication on acceptance

- support for research data, including large and complex data types

- gold Open Access which fosters wider collaboration and increased citations

- maximum visibility for your research: over $100 \mathrm{M}$ website views per year

At $\mathrm{BMC}$, research is always in progress.

Learn more biomedcentral.com/submissions 